Théorie des groupes/Group theory

\title{
Some extremely amenable groups
}

\author{
Thierry Giordano and Vladimir Pestov
}

T.G.: Department of Mathematics and Statistics, University of Ottawa, Ottawa, Ontario, K1N 6N5, Canada.

E-mail: giordano@science.uottawa.ca

URL: http://www.science.uottawa.ca/mathstat/proff/giordano.htm

V.P.: $\quad$ School of Mathematical and Computing Sciences, Victoria University of Wellington, P.O. Box 600, Wellington, New Zealand.

E-mail: vova@mcs.vuw.ac.nz

URL: http://www.mcs.vuw.ac.nz/ vova

\begin{abstract}
A topological group $G$ is extremely amenable if every continuous action of $G$ on a compact space has a fixed point. Using the concentration of measure techniques developed by Gromov and Milman, we prove that the group of automorphisms of a Lebesgue space with a non-atomic measure is extremely amenable with the weak topology but not with the uniform one. Strengthening a de la Harpe's result, we show that a von Neumann algebra is approximately finite-dimensional if and only if its unitary group with the strong topology is the product of an extremely amenable group with a compact group.
\end{abstract}

\section{Quelques groupes extrêmement moyennables}

Résumé - Un groupe topologique G est extrêmement moyennable si toute action continue de $G$ sur un espace compact possède un point fixe. En utilisant les techniques de concentration de mesure développées par Gromov et Milman, nous démontrons que le groupe des automorphismes d'un espace de Lebesgue avec une mesure diffuse est extrêmement moyennable s'il est muni de la topologie faible, mais ne l'est pas avec la topologie uniforme. Si $M$ est une algèbre de von Neumann, nous montrons en utilisant un résultat de $P$. de la Harpe que $M$ est approximativement de dimension finie si et seulement si son groupe unitaire (muni de la topologie forte) est le produit d'un groupe compact et d'un groupe extrêmement moyennable.

\footnotetext{
${ }^{0}$ Nom de la personne qui doit corriger les épreuves: Thierry Giordano Addresse: Département de mathématiques et statistique, Université d'Ottawa, 585 King Edward, Ottawa, Ontario, K1N 6N5, Canada $\mathrm{N}^{\circ}$ de téléphone: +1-613-562-5800 ext. 3514. $\mathrm{N}^{\circ}$ de télécopieur: +1-613-562-5776. Courier électronique: giordano@science.uottawa.ca
} 
Version française abrégée. - Un groupe topologique dont toute action continue sur un espace compact possède un point fixe est dit extrêmement moyennable [6]. Un tel groupe non-trivial est moyennable, mais n'est jamais localement compact [23].

Alors que les premiers exemples de tels groupes ont été difficiles à trouver [13, 1], il a été récemment démontré que de nombreux groupes "de dimensions infinies" sont extrêmement moyennables [8, 4, 17, 19]. Les preuves de ces résultats utilisent souvent les techniques de concentration de mesure dans des espaces de grandes dimensions.

Rappelons qu'un mm-espace $(X, d, \mu)$ est la donnée d'un espace métrique $(X, d)$ muni d'une mesure de probabilité $\mu$. Une suite généralisée $\left(X_{\alpha}, d_{\alpha}, \mu_{\alpha}\right)$ de $m m$-espaces est dite de Lévy si pout tout $\epsilon>0$ et toute famille d'ensembles boréliens $A_{\alpha} \subset X_{\alpha}$ avec $\liminf \mu_{\alpha}\left(A_{\alpha}\right)>0$, on a $\lim \mu_{\alpha}\left(\left(A_{\alpha}\right)_{\epsilon}\right)=1$, où $A_{\epsilon}$ dénote le $\epsilon$-voisinage de $A$.

Exemples.

1. Les groupes de permutations $S_{n}$ munis de la distance de Hamming et de la mesure uniforme (Maurey [14]).

2. Les groupes orthogonaux $S O(n)$ ou les groupes unitaires $S U(n)$ avec la distance induite par la norme de Hilbert-Schmidt et la mesure de Haar normalisée (Gromov et Milman [8]).

Un groupe topologique métrisable est un groupe de Lévy s'il existe une suite généralisée $\left(G_{\alpha}\right)_{\alpha \in A}$ de sous-groupes compacts de $G$ telle que

1. La suite $\left(G, d, \mu_{\alpha}\right)$ est de Lévy, où $d$ dénote une distance invariante à droite qui induit la topologie de $G$ et où $\mu_{\alpha}$ la mesure de Haar normalisée sur $G_{\alpha}$.

2. Pour tout ensemble fini $g_{1}, g_{2}, \cdots, g_{N}$ de $G$ et tout $\epsilon>0$, il existe $\alpha \in A$ tel que, pour tout $\beta \geq \alpha, d\left(g_{i}, G_{\beta}\right)<\epsilon$ pour $1 \leq i \leq N$.

Généralisant légèrement le résultat de [8] (cf. [4] et [19]), nous avons

Théorème. - Un groupe de Lévy est extrêmement moyennable.

Soit $(X, \mu)$ un espace borélien standard muni d'une mesure de probabilité diffuse. Notons $\operatorname{Aut}^{*}(X, \mu)$ (resp. Aut $(X, \mu)$ ) le groupe des automorphismes mesurables nonsinguliers préservant la classe de la mesure $\mu$ (resp. préservant $\mu$ ), muni de la topologie forte.

Théorème. - Tant $\operatorname{Aut}^{*}(X, \mu)$ que Aut $(X, \mu)$ sont des groupes de Lévy et donc sont extrêmement moyennables.

Corollaire. - Si $\mu$ est une mesure $\sigma$-finie, Aut $(X, \mu)$ est extrêmement moyennable.

Par contre, Aut $(X, \mu)$ muni de la topologie uniforme n'est pas extrêmement moyennable, que $\mu$ soit une mesure non-atomique finie ou infinie.

Pour une algèbre de von Neumann $M$, notons $U(M)_{s}$ son groupe unitaire muni de la topologie $\sigma\left(M, M_{*}\right)$. Nous avons alors les deux résultats suivants.

Théorème. - Soit $M$ une algèbre de von Neumann sans partie discrète finie. Alors 
$M$ est approximativement de dimension finie si et seulement si $U(M)_{s}$ est extrêmement moyennable.

Corollaire. - Une algèbre de von Neumann est approximativement de dimension finie si et seulement si son groupe unitaire $U(M)_{s}$ est le produit direct d'un groupe compact et d'un groupe extrêmement moyennable.

Un groupe topologique $G$ est fortement moyennable si toute action continue proximale de $G$ sur un espace compact possède un point fixe [5]. Utilisant un argument semblable à celui de [16] (Thm. 2), nous obtenons

Corollaire. - Une $C^{*}$-algèbre $A$ est nucléaire si et seulement si son groupe unitaire $U(A)$, muni de la topologie $\sigma\left(A, A^{*}\right)$ (induite) faible, est fortement moyennable.

§0. Introduction. Following [6] and [4], we call a topological group whose each continuous action on a compact Hausdorff space has a fixed point, extremely amenable or having the fixed point on compacta property. Since such a group satisfies Day's fixed point property, it is amenable. At the same time, an extremely amenable group is never locally compact, by this result of Veech [23] (cf. also [20]): every locally compact group $G$ acts freely on a suitable compact space. The first examples were difficult to find [13, [1]. However, recently many 'infinite-dimensional' groups were shown to be extremely amenable: the unitary group $U(\mathcal{H})_{s}$ of an infinite-dimensional Hilbert space with the strong operator topology (Gromov and Milman [8]), the group $L_{1}(X, U(1))$ of measurable maps from a non-atomic standard Borel space to the circle group with the topology of convergence in measure (Glasner [4] and Furstenberg and Weiss, unpublished), the orientation-preserving homeomorphism groups Homeo $+(\mathbb{I})$ and $\mathrm{Homeo}_{+}(\mathbb{R})$ [17], the group of isometries of the Urysohn metric space [19]. Most of the proofs use the technique of concentration of measure on high-dimensional structures.

If $X$ is a standard Borel space endowed with a measure $\mu$, we denote by $\operatorname{Aut}(X, \mu)$ (resp. Aut $\left.{ }^{*}(X, \mu)\right)$ the group of all measure (resp. measure class) preserving transformations of $(X, \mu)$ equipped with the weak topology. In this Note we show that, if $\mu$ is a non-atomic sigma-finite (resp. finite) measure, the groups $\operatorname{Aut}(X, \mu)$ and $\operatorname{Aut}^{*}(X, \mu)$ are extremely amenable. With the uniform topology the former group is shown to be non-amenable. Strengthening a de la Harpe's result [12], we show that a von Neumann algebra $M$ is approximately finite-dimensional (AFD) if and only if its unitary group $U(M)_{s}$ with the strong topology is isomorphic to the product of an extremely amenable group with a compact one. As a corollary, we get a new characterization of nuclear $C^{*}$-algebras. 


\section{§1. Lévy families and groups.}

Definition $1.1(\| 7,15])$. An $m m$-space is a triple $(X, d, \mu)$, where $d$ is a metric on a set $X$ and $\mu$ is a probability measure on $(X, d)$. A net $\left(X_{\alpha}, d_{\alpha}, \mu_{\alpha}\right)$ of $m m$-spaces forms a Lévy family if, whenever $A_{\alpha} \subseteq X_{\alpha}$ are Borel subsets with liminf $\mu_{\alpha}\left(A_{\alpha}\right)>0$, for each $\epsilon>0 \lim \mu_{\alpha}\left(\left(A_{\alpha}\right)_{\epsilon}\right)=1$. (Here $A_{\epsilon}$ denotes the $\epsilon$-neighbourhood of a set $A$.)

1.2. Examples of Lévy families. 1. (Maurey [14].) The permutation groups $S_{n}$ of rank $n \geq 1$, equipped with the uniform measure and the Hamming distance,

$$
d_{n}(\sigma, \tau)=\frac{1}{n}|\{i: \sigma(i) \neq \tau(i) \cdot\}|
$$

2. (Gromov and Milman [8].) The special orthogonal groups $S O(n)$ (or the special unitary groups $S U(n)), n \geq 1$, with the Hilbert-Schmidt operator metric and the normalized Haar measure.

The following definition and result slightly generalize those due to Gromov and Milman [8] (cf. [4] and [19]).

Definition 1.3. A metrizable topological group $G$ is a Lévy group if there is a net $\left(G_{\alpha}\right)_{\alpha \in A}$ of compact subgroups of $G$ with the following properties:

1. The family of $m m$-spaces $\left(G, d, \mu_{\alpha}\right)$ is Lévy, where $d$ is any compatible rightinvariant metric on $G$ and $\mu_{\alpha}$ denotes the normalized Haar measure on $G_{\alpha}$.

2. For every finite collection $g_{1}, g_{2}, \ldots, g_{N} \in G, N \in \mathbb{N}$, and every $\epsilon>0$ there is an $\alpha \in A$ with the property: for all $\beta \geq \alpha$ and $i=1,2, \ldots, N, d\left(g_{i}, G_{\beta}\right)<\epsilon$.

Theorem 1.4. Every Lévy group is extremely amenable.

§2. Automorphism groups of a Lebesgue space. Let $X$ be a standard Borel space and let $\mu$ be a non-atomic probability measure on $X$. To every non-singular Borel automorphism $T$ of $(X, \mu)$ one associates a linear isometry $\Phi(T)$ of $L^{2}(X, \mu)$,

$$
\Phi(T) f(x)=\left(\frac{d \mu \circ T^{-1}}{d \mu}(x)\right)^{\frac{1}{2}} f\left(T^{-1} x\right), \text { for } x \in X .
$$

The strong operator topology on the unitary group of $L^{2}(X, \mu)$ induces a Polish group topology on $\operatorname{Aut}^{*}(X, \mu)$ (and also on $\operatorname{Aut}(X, \mu)$ ), called the strong topology. A finer group topology, called the uniform topology, is determined on both groups by the metric

$$
d_{u}(T, S)=\mu(\{x \in X: T(x) \neq S(x)\}) .
$$

Theorem 2.1. The group Aut $(X, \mu)$ of all measure-preserving automorphisms of a non-atomic standard Borel space $X$, equipped with the strong topology, is a Lévy group.

Proof. We identify $(X, \mu)$ with $([0,1], \lambda)$, where $\lambda$ is the Lebesgue measure, and $S_{2^{n}}$ with the subgroup of measure-preserving automorphisms of $[0,1]$ mapping each dyadic interval of rank $n$ onto a dyadic interval of rank $n$ via a translation. According to the 
Weak Approximation Theorem (cf. e.g. [10], pp. 65-68), the union of the increasing sequence of such groups is everywhere dense in Aut $([0,1], \lambda)$. The restriction of the uniform metric $d_{u}$ to each $S_{2^{n}}$ is the Hamming distance (1). Now the proof follows from Maurey's result (1.2.(1)) and Theorem 1.4.

Corollary 2.2. The group Aut $(X)$ of all measure-preserving automorphisms of a standard sigma-finite measure space $(X, \mu)$, equipped with the strong topology, is extremely amenable.

Proof. For $\mu$ finite the result follows from Theorems 2.1 and 1.4, while for $\mu$ sigmafinite one approximates the group $\operatorname{Aut}(X, \mu)$ with groups of finite measure-preserving automorphisms and uses a standard compactness argument.

Denote by $\operatorname{Aut}(X, \mu)_{u}$ the group Aut $(X, \mu)$ equipped with the uniform topology.

Theorem 2.3. The group Aut $(X, \mu)_{u}$ is not amenable, where $\mu$ is either a finite or a sigma-finite non-atomic measure.

Proof. Let $\mu$ be an invariant probability measure on $X=\operatorname{SL}(3, \mathbb{R}) / \operatorname{SL}(3, \mathbb{Z})$. For a Borel set $A \subseteq X$ of measure $\frac{1}{2}$, the function $h_{A}=\chi_{A}-\chi_{A^{c}}$ belongs to the unit sphere $\mathbb{S}_{0}$ of $L_{0}^{2}(X, \mu)=\left\{f \in L^{2}(X, \mu): \int_{X} f d \mu=0\right\}$. There is a $G$-equivariant positive linear operator of norm 1 from the space $\mathrm{UCB}\left(\mathbb{S}_{0}\right)$ of uniformly continuous bounded functions on $\mathbb{S}_{0}$ to the space of right uniformly continuous bounded functions on $\operatorname{Aut}(X, \mu)$ (with the left action of the group), under which a $\varphi \in \mathrm{UCB}\left(\mathbb{S}_{0}\right)$ goes to the function $\operatorname{Aut}(X, \mu) \ni T \mapsto \varphi\left(\Phi(T) h_{A}\right)$. (Cf. formula (2).) If Aut $(X, \mu)_{u}$ were amenable, there would exist an $\operatorname{Aut}(X, \mu)$-invariant mean on $\operatorname{UCB}\left(\mathbb{S}_{0}\right)$, which would imply (Prop. 3.1 in $[18]$ ) that the representation of $\operatorname{SL}(3, \mathbb{R})$ in $L_{0}^{2}(X, \mu)$ is amenable in the sense of Bekka, and by [2], Remark 5.10, would have a non-zero invariant vector, a contradiction. The infinite case is settled by an argument similar to one in 11].

Replacing the Weak Approximation Theorem by its generalization based on a result of Tulcea [22], we obtain the following result.

Theorem 2.4. The group $\operatorname{Aut}^{*}(X, \mu)$ of all measure class preserving automorphisms of a non-atomic standard Borel space $(X, \mu)$, equipped with the strong topology, is a Lévy group and therefore extremely amenable.

$\S 3$. Unitary groups of approximately finite dimensional von Neumann algebras. If $M$ is a von Neumann algebra, we denote by $U(M)_{s}$ its unitary group endowed with the $\sigma\left(M, M_{*}\right)$-topology. If $M$ is acting on a Hilbert space $\mathcal{H}$, this topology on $U(M)$ coincides with the strong operator topology.

Theorem 3.1. Let $M$ be a von Neumann algebra without finite atomic part. Then $M$ is AFD if and only if its unitary group $U(M)_{s}$ is extremely amenable.

Proof. Sufficiency follows from de la Harpe's result [12], in which the separability assumption is not essential [9]. Necessity is a direct consequence of the following four Lemmas. 
Lemma 3.2. The direct product of a family of extremely amenable groups, equipped with the product topology, is extremely amenable.

Using the structure of type I von Neumann algebras and [19], Thm. 2.2, we have

Lemma 3.3. If $M$ is a finite non-atomic type I von Neumann algebra, then $U(M)_{s}$ is extremely amenable.

Lemma 3.4. If $M$ is a finite continuous $A F D$ von Neumann algebra, then $U(M)_{s}$ is extremely amenable.

Proof. Follows from a generalization of a result by Glasner-Furstenberg-Weiss (19, Thm. 2.2).

Lemma 3.5. Let $M$ be a properly infinite AFD von Neumann algebra. Then $U(M)_{s}$ is extremely amenable.

Proof. By Elliott's results [3], we can assume that $M$ has a separable predual and is approximated by an increasing sequence of finite-dimensional factors. It follows that $U(M)_{s}$ is a Lévy group.

Corollary 3.6. A von Neumann algebra $M$ is approximately finite dimensional if and only if its unitary group, $U(M)_{s}$, endowed with the strong operator topology, is the product of a compact group and an extremely amenable group.

A topological group $G$ is called strongly amenable if every continuous proximal action of $G$ on a compact space has a fixed point [5]. By using an argument similar to that in [16] (Thm. 2), we obtain the following.

Corollary 3.7. $A C^{*}$-algebra $A$ is nuclear if and only if its unitary group $U(A)$, equipped with the topology $\sigma\left(A, A^{*}\right)$, is strongly amenable.

Acknowledgements. The authors gratefully acknowledge support from the Swiss National Science Foundation and express their gratitude to Professor Pierre de la Harpe for his hospitality at the Université de Genève. T.G. was also partially supported by an NSREC operating grant, as well as was V.P. during a visit to the University of Ottawa. The research of V.P. was also supported by the VUW Research Development Fund and by a Marsden Fund grant of the Royal Society of New Zealand.

\section{REFERENCES}

[1] Banaszczyk W., On the existence of exotic Banach-Lie groups, Math. Ann. 264 (1983), 485-493.

[2] Bekka M.E.B., Amenable unitary representations of locally compact groups, Invent. Math. 100 (1990), 383-401.

[3] Elliott G.A., On approximately finite-dimensional von Neumann algebras. II, Canad. Math. Bull. 21 (1978), 415-418.

[4] Glasner S., On minimal actions of Polish groups, Top. Appl. 85 (1998), 119-125.

[5] Glasner S., Proximal Flows, Lecture Notes Math. 517, Springer-Verlag, Berlin-New York, 1976. 
[6] Granirer E., Extremely amenable semigroups 2, Math. Scand. 20 (1967), 93-113.

[7] Gromov M., Metric Structures for Riemannian and Non-Riemannian Spaces, Progress in Mathematics 152, Birkhäuser Verlag, 1999.

[8] Gromov M., Milman V.D., A topological application of the isoperimetric inequality, Amer. J. Math. 105 (1983), 843-854.

[9] Haagerup U., All nuclear $C^{*}$-algebras are amenable, Invent. Math. 74 (1983), 305-319.

[10] Halmos, P.R. Lectures on Ergodic Theory, Chelsea Publ. Co., New York, 1960.

[11] de la Harpe P., Moyennabilité de quelques groupes topologiques de dimension infinie, C.R. Acad. Sci. Paris, Sér. A 277 (1973), 1037-1040.

[12] de la Harpe P., Moyennabilité du groupe unitaire et propriété $P$ de Schwartz des algèbres de von Neumann, in: Algèbres d'opérateurs (Sém., Les Plans-sur-Bex, 1978), pp. 220-227, Lecture Notes in Math. 725, Springer-Verlag, Berlin, 1979.

[13] Herer W., Christensen J.P.R., On the existence of pathological submeasures and the construction of exotic topological groups, Math. Ann. 213 (1975), 203-210.

[14] Maurey B., Constructions de suites symétriques, C.R. Acad. Sci. Paris, Sér. A-B 288 (1979), $679-681$.

[15] Milman V.D., The heritage of P.Lévy in geometric functional analysis, Astérisque 157-158 (1988), 273-301.

[16] Paterson A.L.T., Nuclear $C^{*}$-algebras have amenable unitary groups, Proc. Amer. Math. Soc. 114 (1992), 719-721.

[17] Pestov V.G., On free actions, minimal flows, and a problem by Ellis, Trans. Amer. Math. Soc. 350 (1998), 4149-4165.

[18] Pestov V.G., Amenable representations and dynamics of the unit sphere in an infinitedimensional Hilbert space, Geom. Funct. Anal. 10 (2000), 1171-1201.

[19] Pestov V., Ramsey-Milman phenomenon, Urysohn metric spaces, and extremely amenable groups. - Israel J. Math., to appear.

E-print: http://arXiv.org/abs/math.FA/0004010

[20] Pym J., A note on $G^{\text {LUC }}$ and Veech's theorem, Semigroup Forum 59 (1999), 171-174.

[21] Rothman, S., The von Neumann kernel and minimally almost periodic groups, Trans. Amer. Math. Soc. 259 (1980), 401-421.

[22] Tulcea A.I., On the category of certain classes of transformations in ergodic theory, Trans. Amer. Math. Soc. 114 (1965), 261-279.

[23] Veech W.A., Topological dynamics, Bull. Amer. Math. Soc. 83 (1977), 775-830. 